\title{
Факторы, определяющие
}

\section{избыточную доходность}

\section{портфеля ценных бумаг паевых}

\section{инвестиционных фондов}

\author{
Александра Владимировна Галанова \\ Кандидат экономических наук, доцент школы финансов, \\ Национальный исследовательский университет «Высшая школа экономики» \\ Москва, ул. Мясницкая, д. 20 \\ E-mail: agalanova@hse.ru
}

\section{Валерия Валериевна Дукова}

Стажер-исследователь

Национальный исследовательский университет «Высшая школа экономики»

Москва, ул. Мясницкая, д. 20

E-mail: vdukova@hse.ru

\begin{abstract}
Аннотация
В статье предлагается расширение традиционного подхода к выявлению экономических факторов, влияющих на избыточную доходность российских паевых инвестиционных фондов (ПИФ). Цель исследования - установление факторов, воздействующих на избыточную доходность фондов как в агрегированном виде (для российского рынка ПИФов в целом, отдельных типов фондов и направлений их деятельности), так и для конкретных ПИФов (значимость факторов для каждого фонда в отдельности). К наиболее распространенной схеме анализа влияния факторов на избыточную доходность фондов (оценка регрессионными методами модели ценообразования с дальнейшим включением коэффициента альфа из оцененной модели в качестве скорректированной на риск доходности в модель с микро- и макроэкономическими характеристиками фонда) были добавлены: во-первых, анализ моментум-эффекта (простого и скорректированного на риск), который включается в модель Кархарта; во-вторых, тестирование гипотез о значимости коэффициентов бутстрап-методом, что, в частности, позволяет рассматривать избыточную доходность конкретных, отдельно взятых фондов. Были проанализированы данные по 667 российским паевым инвестиционным фондам за период с 2000 по 2016 г. В результате исследования были выявлены следующие факты. При выборе российских ПИФов можно ориентироваться на исторические данные (работает фактор моментума) (оптимальный период - три месяца, хотя в последнее время лучше работает стратегия в 12 месяцев). Корректировка на риск улучшает результаты (лучше всего проявили себя меры Трейнора и Шарпа). Коэффициент а Кархарта значим только у 7\% фондов выборки - не имеет смысла включать его в дальнейший анализ для абсолютно всех фондов. При оценке микроэкономических факторов фондов целесообразно разделить выборку на ПИФы, для которых избыточная доходность задается коэффициентом $\alpha$, и фонды, у которых избыточная доходность определяется относительно бенчмарка (индекс ММВБ), что заметно улучшает получаемые результаты и повышает качество модели.
\end{abstract}

Ключевые слова: паевой инвестиционный фонд (ПИФ), избыточная доходность, бенчмарк, критерии эффективности инвестиций, риск, эффект моментума, метод бутстрап

JEL: G11, G24, G32, G34 


\section{Введение}

Паевые инвестиционные фонды (ПИФы) являются одним из наиболее перспективных инструментов аккумулирования частных сбережений, однако их потенциал еще слабо используется в России. Так, согласно имеющимся данным, «сегодня доля средств частных инвесторов в российских ПИФах составляет 0,2\% ВВП, ... в западных европейских странах - более $42 \% .$. Для того чтобы население начало инвестировать денежные средства в ПИФы, требуется доход на семью в размере 25-30 тыс. долларов в год» [1, с. 18]. Однако российские ПИФы все же представляют значительный интерес, так как, во-первых, имеет место постепенный рост денежных доходов населения, a, во-вторых, они, несомненно, полезны для жителей больших городов, уровень оплаты труда в которых превышает средний ее уровень по стране.

Цель исследования - выявление факторов, влияющих на избыточную доходность фондов как в агрегированном виде (для российского рынка ПИФов в целом, отдельных типов фондов и направлений их деятельности), так и для конкретных ПИФов (значимость факторов для каждого фонда в отдельности) путем использования авторского подхода к их отбору.

Исследование включает следующую последовательность шагов:

1) выбор бенчмарка для определения рыночной и безрисковой доходности;

2) оценка регрессионными методами модели ценообразования (CAPM, многофакторные модели);

3) включение коэффициента из оцененной модели (в основном модели Кархарта) в качестве скорректированной на риск доходности в регрессионную модель с микро- и макроэкономическими характеристиками фонда.

На шаге 2 проводился анализ фактора моментума ${ }^{1}$, скорректированного на риск, с целью понять, можно ли при выборе российских ПИФов ориентироваться на результаты их прошлой деятельности, и если да, то за какой период.

Затем оценивается значимость коэффициента методом бутстрап (bootstrap), использование которого представляется необходимым ввиду некорректности применения обычных способов тестирования гипотез.

Одновременно осуществляется тестирование гипотез о значимости коэффициентов методом бутстрап, что позволяет выявить ПИФы, для которых значим тот или иной фактор, и направление его влияния на избыточную доходность.

Обычно для сравнительной оценки ПИФов используют коэффициенты Шарпа, Сортино, $\mathrm{VaR}$, вола- тильность (стандартное отклонение), $R^{2}, \alpha-$ и $\beta$-коэффициенты. Однако простое использование данных коэффициентов, как показывает опыт выбора ПИФов для инвестирования, часто не дает желаемых результатов. Поэтому имеющуюся информацию приходится преобразовывать в целях усиления ее прогнозной силы.

\section{Результаты эмпирического тестирования базовых моделей на разных рынках}

Базовые теоретические модели могут использоваться для определения факторов, влияющих на избыточную доходность фондов сами по себе, а также применяться для оценки избыточной доходности - независимой переменной в регрессионном анализе с характеристиками фондов, макроэкономическими факторами и т.д. В связи с этим имеющиеся эмпирические работы можно разделить на две группы:

1) статьи, в которых предлагается тестирование моделей САРМ, Фамы - Френча, Кархарта (условных и безусловных) с целью оценить значимость и направление коэффициентов перед факторами, описательную силу модели на исследуемом рынке. Затем, как правило, делается вывод о том, переигрывают ли рынок фонды в данной стране или проигрывают рынку. Краткий обзор статей этой категории в систематизированном виде можно представить в виде табл. 1;

2) статьи, авторы которых используют модели ценообразования для определения избыточной доходности фонда, после чего включают этот показатель в модель регрессионного анализа с различными микро- и макроэкономическими факторами (используются как поперечные, так и панельные данные).

В большинстве рассмотренных работ в качестве зависимой переменной принимается коэффициент из модели CAPM и ее модификаций (в основном из четырехфакторной модели Кархарта). Считается, что этот коэффициент позволяет учесть прирост доходности за счет навыков управляющих, а в случае многофакторных моделей - с корректировкой на влияние факторов размера, стоимости и (или) моментума.

Среди независимых переменных выделяются такие факторы, как размер фонда (аппроксимируемый объемами СЧА); размер «семьи», в которую входит фонд (количество фондов в управляющей компании - УК); издержки (скидки и вознаграждения) инвестора; различные макроэкономические факторы, отвечающие за межстрановые различия, и т.п.

\footnotetext{
${ }^{1}$ Моментум-эффект - ценовая аномалия на фондовом рынке, при которой портфели, сформированные по принципу результатов прошлой деятельности (по ранее наблюдавшимся доходностям или другим индикаторам эффективности инвестирования), показывают избыточную доходность по отношению к заданному бенчмарку.
} 
Таблица 1. Обзор эмпирических исследований, посвященных определению факторов, влияющих на избыточную доходность фондов на разных рынках, путем оценки одно/многофакторных моделей

\begin{tabular}{|c|c|c|c|c|c|c|c|c|c|c|}
\hline \multirow{2}{*}{ Оцениваемая модель } & \multirow{2}{*}{ Тип модели } & \multicolumn{5}{|c|}{ Значимость и направление влияния коэффициентов перед факторами* } & \multirow{2}{*}{$\begin{array}{l}\text { Описательная сила } \\
\text { модели }\end{array}$} & \multirow{2}{*}{$\begin{array}{l}\text { Данные и период } \\
\text { времени }\end{array}$} & \multirow{2}{*}{$\begin{array}{l}\text { Фонды переигрывают } \\
\text { рынок/проигрывают } \\
\text { рынку }\end{array}$} & \multirow{2}{*}{ Авторь } \\
\hline & & a & $\boldsymbol{R}_{m}-\boldsymbol{R}_{f}$ & $S M B$ & $H M L$ & MOM & & & & \\
\hline CAPM & Безусловная & $\ll-»$ & $\ll+»^{*}$ & & & & Высокая & \multirow{3}{*}{$\begin{array}{l}\text { Этические фонды } \\
\text { в Германии, } \\
\text { Великобритании, } \\
\text { США } \\
\text { (1995-2000) }\end{array}$} & \multirow{3}{*}{$\begin{array}{l}\text { В Германии и США - } \\
\text { проигрывают, } \\
\text { в Великобритании - } \\
\text { переигрывают }\end{array}$} & \multirow{3}{*}[2]{} \\
\hline \multirow{2}{*}{$\begin{array}{l}\text { Четырехфакторная модель } \\
\text { Кархарта }\end{array}$} & Безусловная & $«-»$ & $《+\rangle^{*}$ & $《+\rangle^{*}$ & $\ll-»$ & $«+»^{*}$ & Высокая & & & \\
\hline & Условная & $\ll-»^{*}$ & $«+»^{*}$ & $«+»^{*}$ & $«-»$ & $«+»^{*}$ & Высокая & & & \\
\hline CAPM & Безусловная & $«+»$ & $«+\rangle^{*}$ & & & & Средняя & \multirow{3}{*}{$\begin{array}{l}\text { Исламские фонды } \\
\text { в } 20 \text { странах } \\
\text { (1990-2009) }\end{array}$} & \multirow{3}{*}{ Переигрывают } & \multirow{3}{*}{ [3] } \\
\hline \multirow{2}{*}{$\begin{array}{l}\text { Четырехфакторная модель } \\
\text { Кархарта }\end{array}$} & Безусловная & $«-»$ & $\ll+»^{*}$ & $《+\rangle^{*}$ & $《-\rangle^{*}$ & $«-»$ & Средняя & & & \\
\hline & Условная & $《-»^{*}$ & $《+\rangle^{*}$ & $《+\rangle^{*}$ & $《-\rangle^{*}$ & $\ll+\rangle^{*}$ & Высокая & & & \\
\hline $\begin{array}{l}\text { Трехфакторная модель } \\
\text { Фамы -Френча }\end{array}$ & Безусловная & $《-»^{*}$ & $«+»$ & «+» & «-» & & Средняя & $\begin{array}{l}\text { Фонды облигаций } \\
\text { США } \\
\text { (1991-1998) }\end{array}$ & Проигрывают & {$[4]$} \\
\hline CAPM & Безусловная & $《-\rangle^{*}$ & $《+\rangle^{*}$ & & & & Высокая & \multirow{2}{*}{$\begin{array}{l}\text { Открытые фонды } \\
\text { США } \\
\text { (1994-1996) }\end{array}$} & \multirow[b]{2}{*}{ Переигрывают } & \multirow{2}{*}{ [5] } \\
\hline $\begin{array}{l}\text { Четырехфакторная модель } \\
\text { Кархарта }\end{array}$ & Безусловная & «-» & $\ll+»^{*}$ & $《+\rangle^{*}$ & $《-\rangle^{*}$ & $«+»^{*}$ & Высокая & & & \\
\hline CAPM & Безусловная & $«-»$ & $《+\rangle^{*}$ & & & & Высокая & \multirow{3}{*}{$\begin{array}{l}\text { Фонды США всех } \\
\text { типов } \\
\text { (1984-2003) }\end{array}$} & \multirow{3}{*}{ Переигрывают } & \multirow{3}{*}[6]{} \\
\hline $\begin{array}{l}\text { Трехфакторная модель } \\
\text { Фамы -Френча }\end{array}$ & Безусловная & $«+»$ & $《+»^{*}$ & «+» & & & Средняя & & & \\
\hline $\begin{array}{l}\text { Четырехфакторная модель } \\
\text { Кархарта }\end{array}$ & Безусловная & «-» & $《+\rangle^{*}$ & $«+»$ & $\ll-»$ & & Высокая & & & \\
\hline $\begin{array}{l}\text { Четырехфакторная модель } \\
\text { Кархарта }\end{array}$ & Безусловная & $《-»^{*}$ & $《+\rangle^{*}$ & $《+»^{*}$ & $《-»^{*}$ & $《+\rangle^{*}$ & Средняя & $\begin{array}{l}\text { Открытые фонды } \\
\text { США } \\
\text { (1994-2004) }\end{array}$ & $\begin{array}{l}\text { Зависит от выбранного } \\
\text { бенчмарка } \\
\text { (всего 12) }\end{array}$ & [7] \\
\hline \multirow{3}{*}{$\begin{array}{l}\text { Четырехфакторная модель } \\
\text { Кархарта }\end{array}$} & Безусловная & $《+\rangle^{*}$ & $《+\rangle^{*}$ & $《+\rangle^{*}$ & $《+»^{*}$ & $«+»^{*}$ & Низкая & \multirow{3}{*}{$\begin{array}{l}\text { Открытые фонды } \\
\text { Норвегии } \\
\text { (2000-2010) }\end{array}$} & \multirow{3}{*}{ Нейтральны } & \multirow{3}{*}[8]{} \\
\hline & Условная & $《+»^{*}$ & $《+\rangle^{*}$ & $《+\rangle^{*}$ & $《+»^{*}$ & $«+»^{*}$ & Низкая & & & \\
\hline & Многопериодная & $\langle-\gg /\langle+»$ & $\ll+\rangle^{*}$ & $《+\rangle^{*}$ & $\langle-\gg /\langle+»$ & $《-\gg / \ll+»$ & Средняя & & & \\
\hline $\begin{array}{l}\text { Четырехфакторная модель } \\
\text { Кархарта }\end{array}$ & Безусловная & $《-»^{*}$ & $《+\rangle^{*}$ & «+» & $\ll-»$ & $«-»$ & Средняя & $\begin{array}{l}\text { Интервальные } \\
\text { фонды Швеции } \\
\text { (2005-2015) }\end{array}$ & Переигрывают & [9] \\
\hline
\end{tabular}

* Примечание: «+» - положительное влияние коэффициента; «-» - отрицательное влияние коэффициента; ${ }^{*}$ - коэффициент значим. 
Таблица 2. Обзор эмпирических исследований, посвященных определению факторов, влияющих на избыточную доходность фондов на разных рынках, методами регрессионного анализа

\begin{tabular}{|c|c|c|c|c|c|c|}
\hline \multirow{2}{*}{$\begin{array}{l}\text { Фактор и влияние на избыточную } \\
\text { доходность }\end{array}$} & \multirow[t]{2}{*}{ Объяснение } & \multirow{2}{*}{$\begin{array}{l}\text { Моделирование } \\
\text { избыточной доходности }\end{array}$} & \multicolumn{2}{|c|}{ Регрессионный анализ } & \multirow{2}{*}{$\begin{array}{l}\text { Данные и период } \\
\text { времени }\end{array}$} & \multirow[t]{2}{*}{ Авторы } \\
\hline & & & Зависимые переменныте & Независимые переменные & & \\
\hline \multicolumn{7}{|l|}{ Размер фонда } \\
\hline «+» & \multirow{2}{*}{$\begin{array}{l}\text { У крупных фондов есть возможность } \\
\text { привлечь более квалифицированных } \\
\text { управляющих }\end{array}$} & \multirow{6}{*}{$\begin{array}{l}\text { Доходности индекса ММВБ } \\
\text { (фонды акций и индексные } \\
\text { фонды) и ОФ3 (фонды } \\
\text { облигаций, денежного } \\
\text { рынка), стратегия 50/50 } \\
\text { фонды смешанных } \\
\text { инвестиций; } \\
\text { четырехфакторная модель } \\
\text { Кархарта }\end{array}$} & \multirow{6}{*}{$\begin{array}{l}\text { Избыточная доходность по } \\
\text { отношению к доходностям } \\
\text { индекса ММВБ и ОФЗ, в } \\
\text { зависимости от типа фонда, } \\
\text { альфа из модели Кархарта }\end{array}$} & \multirow{6}{*}{$\begin{array}{l}\text { Объем СЧА, возраст фонда, } \\
\text { баланс продаж, количество } \\
\text { фондов в УК, издержки } \\
\text { инвестора, оборачиваемость } \\
\text { портфеля, бинарная } \\
\text { переменная на тип фонда, } \\
\text { частота цитирования УК в } \\
\text { СМИ }\end{array}$} & \multirow{6}{*}{$\begin{array}{l}\text { Открытые и } \\
\text { интервальные фонды } \\
\text { России } \\
(2007-2012)\end{array}$} & \multirow{6}{*}{$\begin{array}{l}{[10, \text { c. } 543-561 ;} \\
11 ; 12]\end{array}$} \\
\hline $\begin{array}{l}\text { Количество фондов в УК } \\
\text { «» }\end{array}$ & & & & & & \\
\hline $\begin{array}{l}\text { Возраст фонда } \\
\text { «-» для фондов акций и индивидуальных } \\
\text { фондов, «+» для фондов облигаций, } \\
\text { денежного рынка, смешанных инвестиций }\end{array}$ & $\begin{array}{l}\text { Более «молодые» фонды акций выбирают } \\
\text { лучшие стратегии инвестирования, более } \\
\text { «старые» фонды облигаций менее склонны } \\
\text { завышать доходность }\end{array}$ & & & & & \\
\hline $\begin{array}{l}\text { Издержки инвестора } \\
\text { «+» }\end{array}$ & $\begin{array}{l}\text { Расходы на управление растут с } \\
\text { привлечением более профессиональных } \\
\text { сотрудников }\end{array}$ & & & & & \\
\hline Продажа паев через агентов «+» & $\begin{array}{l}\text { К продаже паев через агентов допускаются } \\
\text { только «хорошие» фонды }\end{array}$ & & & & & \\
\hline $\begin{array}{l}\text { Поток денежных средств } \\
\text { (баланс продаж) } \\
\text { «+» }\end{array}$ & $\begin{array}{l}\text { «Эффект умных денег» (зависимость } \\
\text { избыточной доходности от баланса продаж } \\
\text { текущего периода) и «эффект моментума» }\end{array}$ & & & & & \\
\hline $\begin{array}{l}\text { Количество фондов в УК } \\
\text { «» }\end{array}$ & $\begin{array}{l}\text { Внутри «семьи» фондов наблюдаются } \\
\text { «перетоки» средств, если какие-то фонды } \\
\text { становятся проблемными }\end{array}$ & $\begin{array}{l}\text { Мера Шарпа, } \\
\text { альфа Дженсена, индексы } \\
\text { EURIBOR, } \\
\text { MSCI Germany, MSCI } \\
\text { Europe }\end{array}$ & $\begin{array}{l}\text { Избыточная доходность из } \\
\text { модели CAPM, чистые потоки } \\
\text { фонда }\end{array}$ & $\begin{array}{l}\text { Количество фондов в УК, } \\
\text { контрольные переменные на } \\
\text { размер фонда }\end{array}$ & $\begin{array}{l}\text { Фонды Германии всех } \\
\text { типов } \\
\text { (2003-2008) }\end{array}$ & {$[13]$} \\
\hline $\begin{array}{l}\text { Количество фондов в УК } \\
\text { «+» }\end{array}$ & $\begin{array}{l}\text { «фффект масштаба»: большие «семьи» } \\
\text { фондов достигают экономии на издержках } \\
\text { управления и привлечения новых средств }\end{array}$ & $\begin{array}{l}\text { Вероятность повышения/ } \\
\text { понижения доходности } \\
\text { (логит-модель) }\end{array}$ & Средняя доходность & $\begin{array}{l}\text { Объем СЧА, возраст фонда, } \\
\text { сектор, количество фондов } \\
\text { в УК, издержки инвестора, } \\
\text { бинарная переменная - фонд } \\
\text { стоимости }\end{array}$ & $\begin{array}{l}\text { Открытые фонды США } \\
\text { (2002-2006) }\end{array}$ & {$[14]$} \\
\hline $\begin{array}{l}\text { Размер фонда } \\
\ll-»\end{array}$ & $\begin{array}{l}\text { С увеличением размера фонда растут } \\
\text { издержки на поддержание ликвидности } \\
\text { паев }\end{array}$ & $\begin{array}{l}\text { САРМ, } \\
\text { трех- и четырехфакторные } \\
\text { модели }\end{array}$ & $\begin{array}{l}\text { Избыточная доходность из } \\
\text { модели САРM }\end{array}$ & $\begin{array}{l}\text { Объем СЧА, количество } \\
\text { фондов в УК, бинарная } \\
\text { переменная на объект } \\
\text { инвестирования }\end{array}$ & $\begin{array}{l}\text { Открытые фонды США } \\
\text { (1962-1999) }\end{array}$ & {$[15]$} \\
\hline
\end{tabular}




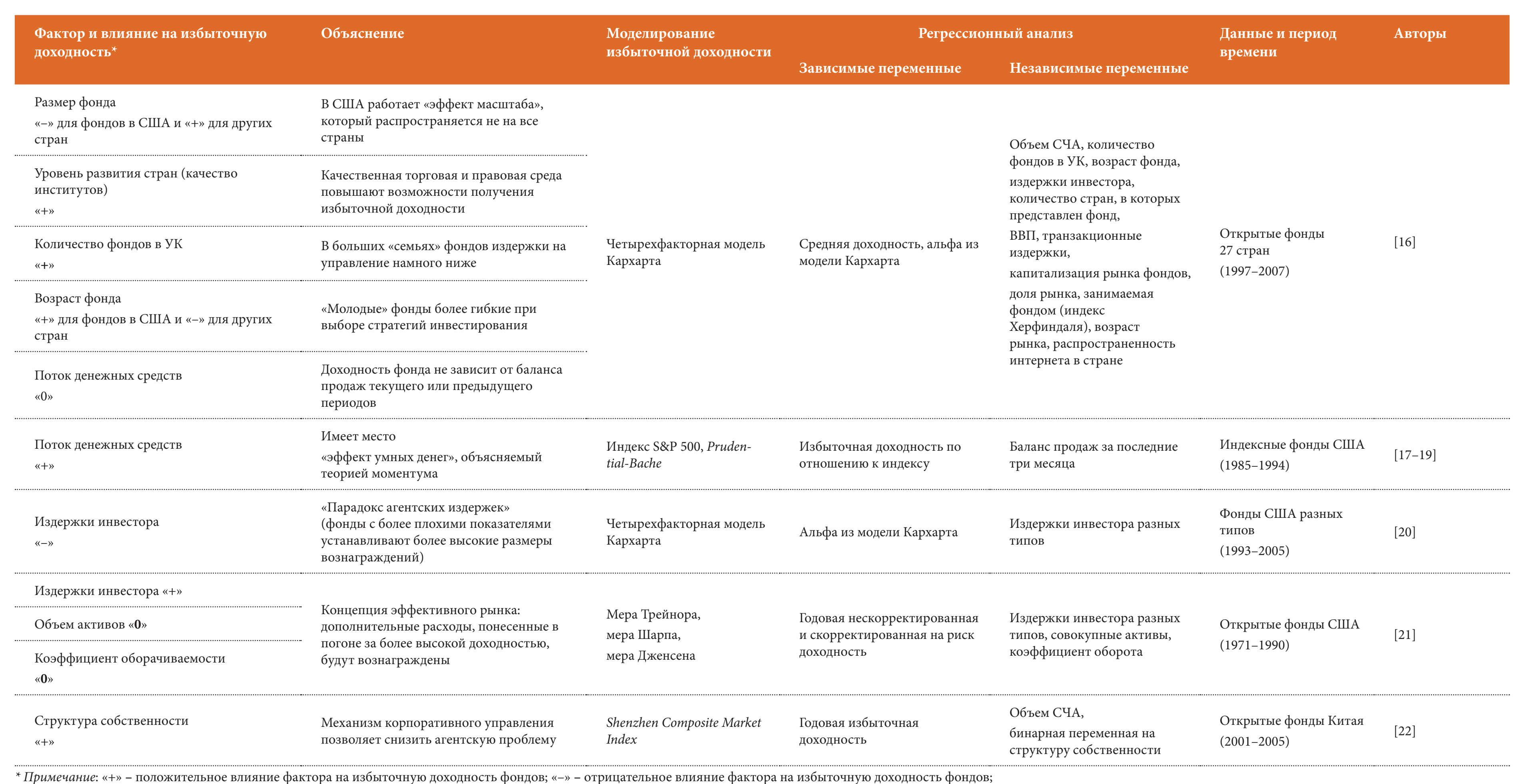

* Примечание: «+» - положительное влияние фактора на избыточную доходность фондов; «-» - отрицательное влияние фактора на избыточную доходность фондов;

«0» - избыточная доходность, не зависящая от данного фактора.

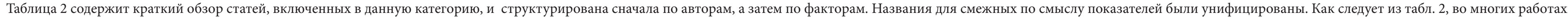
авторы исследовали влияние набора факторов на избыточную доходность и делали выводы. В данном случае были выбраны лишь основные из них, которые учитывались при эмпирическом тестировании факторов. 
Таблица 3. Данные по ряду сопутствующих показателей и характеристик ПИФов

\begin{tabular}{|c|c|}
\hline Данные по управляющим компаниям (УК) & Данные по ПИФам \\
\hline 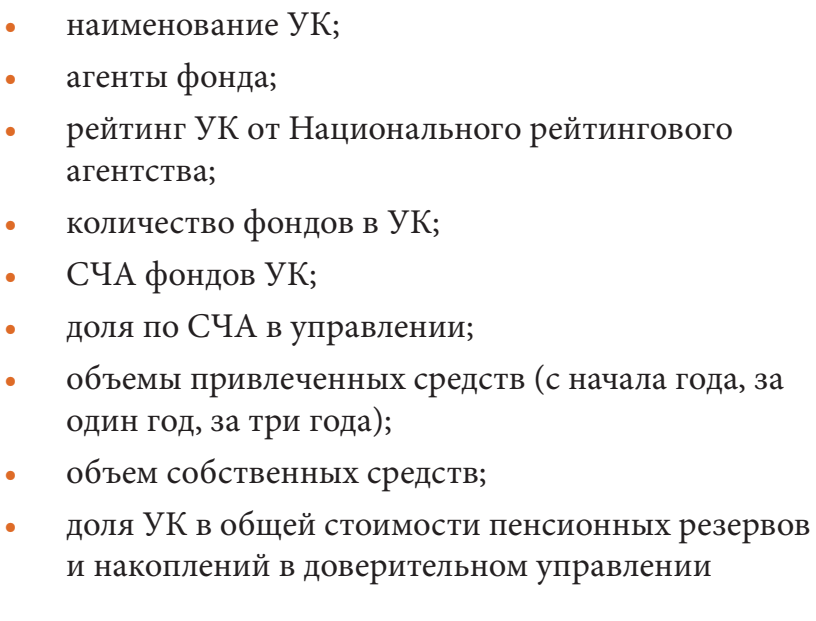 & $\begin{array}{ll}\text { • } & \text { тип фонда; } \\
\text { • } & \text { объект инвестирования; } \\
\text { даты регистрации, начала и окончания } \\
\text { • } \quad \text { колмирования фонда; } \\
\text { - } \quad \text { структура активов; } \\
\text { минимальные суммы первого и последующих } \\
\text { взносов; } \\
\text { надбавки и скидки (общие и УК), в зависимости } \\
\text { от суммы приобретения и срока владения, } \\
\text { соответственно; } \\
\text { вознаграждения (УК, депозитарию и др., прочие } \\
\text { расходы) }\end{array}$ \\
\hline
\end{tabular}

Данные были получены из следующих информационных ресурсов: InvestFunds, НЛУ, Финам, Micex, ЦБ РФ, Росстат, MCSI Barra.

Таблица 4. Классификация паевых инвестиционных фондов в итоговой выборке

\begin{tabular}{|c|c|c|c|}
\hline Тип фонда & Объект инвестирования & Количество фондов & Итого \\
\hline \multirow{6}{*}{$\begin{array}{l}\text { Открытый } \\
\text { (347) }\end{array}$} & Акции & 118 & \multirow{3}{*}{190} \\
\hline & Индекс & 22 & \\
\hline & Фонды & 50 & \\
\hline & Облигации & 73 & \multirow{3}{*}{157} \\
\hline & Денежный рынок & 9 & \\
\hline & Смешанный & 75 & \\
\hline \multirow{3}{*}{$\begin{array}{l}\text { Интервальный } \\
\text { (28) }\end{array}$} & Акции & 13 & 13 \\
\hline & Смешанный & 11 & \multirow{2}{*}{15} \\
\hline & Товарный рынок & 4 & \\
\hline \multirow{6}{*}{$\begin{array}{l}\text { Закрытый } \\
\text { (224) }\end{array}$} & Акции & 15 & 15 \\
\hline & Смешанный & 20 & \multirow{5}{*}{209} \\
\hline & Недвижимость & 141 & \\
\hline & Рентный & 45 & \\
\hline & Ипотека & 2 & \\
\hline & Художественные ценности & 1 & \\
\hline Итого & & 599 & \\
\hline
\end{tabular}

\section{Описание выборки}

При проведении исследования были собраны и обработаны данные по 667 российским паевым инвестиционным фондам за период с января 2000 г. по апрель 2016 г.:

- $\quad$ стоимость пая и СЧА фонда (с дневной периодичностью);
- данные по ряду сопутствующих показателей и характеристик (табл. 3).

В первоначальную выборку входили 347 открытых, 30 интервальных и 290 закрытых ПИФов.

Поскольку применение статистического аппарата требует большого числа наблюдений, то из выборки были исключены все фонды с числом наблюдений менее $90^{2}$.

\footnotetext{
${ }^{2}$ Выбирая минимально допустимое количество наблюдений, мы ориентировались на работу [23], где оно составило 100 наблюдений. Однако был учтен тот факт, что в нашей выборке имеется 140 фондов с количеством наблюдений менее 100 , из которых 70 фондов - с количеством наблюдений от 90 до 100.
} 
Таким образом, в итоговую выборку вошли 599 фондов (347 открытых, 28 интервальных, 224 закрытых).

Регрессионный анализ проводился для подвыборок, сформированных из фондов с разными объектами инвестирования, которые были сгруппированы по уровню рыночного риска (табл. 4). Как видно из табл. 4, несмотря на то, что между 15 закрытыми ПИФами акций и 209 остальными фондами закрытого типа имеется большая разница (по объему под- выборки), мы считаем, что они не сопоставимы по риску, и их нельзя рассматривать как одну (единую) группу.

Гипотеза о нормальности распределения была проверена при помощи теста Харке - Бера, и на уровне значимости 5\% было выявлено всего 14 фондов с доходностью, подчиняющейся нормальному закону распределения (нулевая гипотеза о нормальности распределения не отвергается).

Таблица 5. Фонды с нормальным распределением доходностей на уровне значимости 5\% (итоговая выборка)

\begin{tabular}{|c|c|c|c|}
\hline $\begin{array}{l}\text { Название } \\
\text { фонда }\end{array}$ & $\begin{array}{l}\text { P-value (тест } \\
\text { Харке - Бера) }\end{array}$ & $\begin{array}{l}\text { Название } \\
\text { фонда }\end{array}$ & $\begin{array}{l}P \text {-value (тест } \\
\text { Харке - Бера) }\end{array}$ \\
\hline АК БАРС - СОЗВЕЗДИЕ & 0.5816 & СКМ-Недвижимость & 0.8977 \\
\hline Лидер Девелопмент & 0.7403 & Своя земля - рентный & 0.8465 \\
\hline Атлант & 0.0993 & ДОХОД - Рентная недвижимость & 0.5326 \\
\hline Структурный & 0.1189 & Стратег & 0.2250 \\
\hline СЭМ Эстейт Первый & 0.6336 & Стрит Ритейл & 0.3328 \\
\hline Фонд стратегических инвестиций-3 & 0.0779 & Основная бизнес-модель - Альфа & 0.7549 \\
\hline Четвертый Земельный & 0.3891 & Солид - Недвижимость-2 & 0.3916 \\
\hline
\end{tabular}

\section{Методы исследования}

Расчеты проводились по двум типам моделей:

- $\quad$ тип 1 - многофакторные модели, основанные на модели САРМ (трехфакторная модель Фамы Френча, четырехфакторная модель Кархарта) с факторами размера $(S M B)$, стоимости $(H M L)$, моментума (MOM);

- $\quad$ тип 2 - регрессионные модели с набором факторов - микроэкономических характеристик фондов.

При оценке моделей первого типа применялись методы анализа панельных данных.

Модели второго типа строились на базе поперечных данных:

- $\quad$ во-первых, ввиду наличия данных по микроэкономическим характеристикам фондов только на последний момент времени;

- во-вторых, даже если бы по этим факторам имелись временные ряды тогда, когда для каких-то фондов найдутся статистически-значимые Кархарта, модель типа 2 все равно перейдет в модель анализа поперечных данных.

Модели типа 2 оценивались простым методом наименьших квадратов (МНК).

Для моделей типа 1 применялись три подхода к оценке регрессий по панельным данным:
- $\quad$ построение регрессии по объединенной выборке (pooling): используется при отсутствии значимых различий (неоднородности) между объектами выборки;

- $\quad$ модель с фиксированными эффектами (по типу within): неоднородность данных учитывается рассмотрением фиксированных эффектов путем введения фиктивных переменных для объектов выборки, коэффициенты при которых будут давать оценки индивидуальных эффектов;

- модель со случайными эффектами (random): используется для получения более эффективных оценок (снижаются потери степеней свобод) в случае, если ненаблюдаемые факторы не коррелируют с регрессорами; основывается на выборе случайным образом некоторого количества объектов из большой генеральной совокупности ${ }^{3}$.

При оценке качества регрессионной модели методом МНК одной из предпосылок является нормальное распределение остатков регрессии. К нарушению этой предпосылки могут привести несколько факторов [23]:

- доходность отдельных фондов в выборке может иметь распределение, отличное от нормального;

- ряды доходностей могут быть нестационарными, с автокорреляцией;

- в случайных составляющих доходностей может присутствовать гетероскедастичность.

\footnotetext{
3 Выбор между моделью pooling и моделью within проводится с помощью стандартного F-теста. Выбор между моделью рооling и моделью random проводится с помощью теста Бройша - Пагана (строится на основе метода максимального правдоподобия). Выбор между моделью random и моделью within проводится с помощью статистики Хаусмана путем сравнения оценок внутригрупповой регрессии и регрессии со случайными эффектами.
} 
При наличии этих факторов значимость коэффициентов нельзя проверять стандартными методами. У большинства ПИФов распределение доходностей отличается от нормального. Поэтому для проверки гипотез о значимости коэффициентов приходится использовать метод бутстрап, позволяющий уменьшить разницу между реальной и полученной вероятностью и отвергнуть тестируемую нулевую гипотезу [24-26].

\section{Определение факторов, влияющих на избыточную доходность российских ПИФов}

Эмпирический анализ факторов, влияющих на избыточную доходность российских ПИФов, был проведен в следующей последовательности:

- $\quad$ определение наиболее подходящего бенчмарка избыточной доходности для российских ПИФов;

- $\quad$ тестирование модели Фамы - Френча по дневным данным (факторы размера и стоимости);

- расчет коэффициентов эффективности инвестиционной деятельности фондов (коэффициенты Шарпа, Трейнора, Сортино и т.д.);

- определение фактора моментума, в том числе с поправкой на риск (оцененный коэффициентами Шарпа, Трейнора, Сортино и т.д.);

- оценка четырехфакторной модели Кархарта (с включенным фактором моментума) методами анализа панельных данных (pooling, within, random);

- тестирование гипотезы о значимости коэффициентов из модели Кархарта методом бутстрап;

- $\quad$ выявление фондов, для которых значим коэффициент перед тем или иным фактором из модели Кархарта;

- описание влияния факторов из модели Кархарта $(S M B, H M L, M O M)$ на избыточную доходность фондов;

- $\quad$ в случае если для фонда коэффициент а Кархарта значим, включаем его в качестве зависимой переменной в cross-sectional регрессию с факторами микроэкономическими характеристиками фондов; в противном случае используем избыточную по отношению к бенчмарку доходность;

- описание влияния факторов микроэкономических характеристик фондов на избыточную доходность российских ПИФов.

\section{Выбор бенчмарка для определения избыточной доходности и качество оцениваемой модели}

Бенчмарк ${ }^{4}$ во многом определяет описательную силу оцениваемой модели. Данный показатель можно использовать и самостоятельно, альтернативно применению коэффициентов Трейнора, Шарпа и т.д. для тестирования эффективности деятельности фонда.

Проблема выбора правильного бенчмарка для определения избыточной доходности поднимается многими исследователями. Например, Ипполито [27] и Линч [28] говорили о том, что применение несоответствующего бенчмарка приводит к несостоятельности оцениваемой модели. Другие исследования [29, 30] позволили сделать вывод о том, что результаты моделей, основанных на САРМ, являются смещенными тогда, когда оцениваются фонды с небольшой капитализацией, а в качестве бенчмарка берутся рыночные индексы с большой капитализацией (Benchmark Error).

Ненормальное распределение доходностей бенчмарка или ее нелинейность приводят к смещенным оценкам альфы Дженсена [31]. Соответственно, это ставит под вопрос пригодность альфы Дженсена в качестве показателя избыточной доходности в дальнейшем регрессионном анализе.

В своей работе Элтон и др. [32] показали, как неправильный выбор бенчмарка приводит к тому, что факторы размера и стоимости из моделей Фамы Френча и Кархарта, в действительности оказывающее существенное влияние на избыточную доходность, становятся незначимыми.

На данный момент нет точного метода определения инвестиционного ориентира и правил моделирования бенчмарка, что порождает множество споров. Чаще всего в качестве бенчмарка выбирают рыночные индексы, однако ввиду широкого охвата рынка результаты их применения к отдельному фонду могут оказаться смещенными [33, 34].

В рамках данного исследования в качестве показателя избыточной доходности использовались:

- $\quad$ превышение доходности фонда над доходностью бенчмарка;

- $\quad$ коэффициент из моделей ценообразования (CAPM, многофакторных моделей, как безусловных, так и условных, и т.д.).

Расчеты показывают, что доходность индекса ММВБ в относительно большей степени аппроксимируется нормальным законом распределения, чем любые другие индексы, рассчитываемые на российском фондовом рынке. Кроме того, он рассчитывается на основе рублевых цен, а не цен в долларах США, как индекс РТС. Поэтому в качестве бенчмарка был выбран индекс ММВБ.

${ }^{4}$ Бенчмарк - это индекс или иной показатель, относительно которого рассчитывается избыточная доходность инвестиционного актива. Может интерпретировать как доходность пассивной стратегии (buy-and-hold). 
В проведенных расчетах доходность индекса ММВБ задавала ставку при оценке моделей типа 1 и использовалась в качестве бенчмарка, относительно которого рассчитывается избыточная доходность при оценке моделей типа 2 (для тех фондов, у которых Кархарта окажется незначимой). Доходность ОФЗ будет задавать ставку .

\section{Проверка гипотез на основе тестирования базовых теоретических моделей на данных по российским ПИФам}

Поскольку модель Фамы - Френча не включает фактора моментума и оценивается по дневным данным, отдельно рассматриваем фактор моментума, после чего включаем его в четырехфакторную модель Кархарта (оценивается по месячным данным). Результаты из модели Кархарта используем и в последующих расчетах.

\section{Гипотеза 1: на избыточную доходность российских ПИФов влияют факторы размера и стоимости.}

Оцениваем следующую модель Фамы и Френча:

$$
\begin{aligned}
& R_{i t}-R_{f t}=\alpha_{i}+\beta_{i 1}\left(R_{m t}-R_{f t}\right)+ \\
& +b_{i 2} S M B_{t}+b_{i 3} H M L_{t}+\varepsilon_{i t}
\end{aligned} .
$$

Используем методологию регрессионного анализа панельных данных.

Порядок расчета показателя следующий. На момент времени все фонды ранжируются по показателю размера (СЧА фондов) от большего к меньшему и делятся на две группы с одинаковым количеством фондов («большие» фонды входят в портфели с индексами $B / L, B / M$ и $B / H$; «маленькие» фонды - в портфели $S / L$, $S / M$ и $S / H)$.

Портфель $S M B$ строится для имитации фактора риска, связанного с эффектом размера фондов, и рассчитывается как разность между средневзвешенной доходностью второй и первой квантилей:

$$
\begin{aligned}
& \text { SMB }=\frac{1}{3}\left(\text { Portfolio }_{S / L}+\text { Portfolio }_{S / M}+\text { Portfolio }_{S / H}\right) \\
& -\frac{1}{3}\left(\text { Portfolio }_{B / L}+\text { Portfolio }_{B / M}+\text { Portfolio }_{B / H}\right)
\end{aligned} .
$$

Порядок расчета показателя следующий. Независимо от первой сортировки на момент времени все фонды ранжируются по величине коэффициента $B V / M V$ от меньшего к большему. Значение коэффициента $B V / M V$ рассчитывается путем деления стоимости инвестиционного пая в нулевой момент времени на текущую стоимость пая. Анализируемая выборка делится на три группы: 30, 40 и $30 \%$ соответственно. Фонды с «низким» значением коэффициента $B V / M V$ входят в портфели с индексами $S / L$ и $B / L$, со «средним» значением - в портфели с индексами $S / M$ и $B / M$; с «высоким» - в портфели с индексами $S / H$ и $B / H)$.

Показатель строится для имитации фактора стоимости, связанного с эффектом размера фондов, и рассчитывается как разность между средневзвешенной доходностью третьей и первой квантилей:

$$
\begin{aligned}
& H M L=\frac{1}{2}\left(\text { Portfolio }_{S / H}+\text { Portfolio }_{B / H}\right)- \\
& -\frac{1}{2}\left(\text { Portfolio }_{S / L}+\text { Portfolio }_{B / L}\right)
\end{aligned}
$$

Для определения факторов, влияющих на избыточную доходность разных типов ПИФов, регрессии оцениваются как для целой выборки (всех фондов), так и для групп фондов (табл. 6).

Поскольку зависимая переменная представляет собой панельный набор данных, регрессионные модели оценивались при помощи трех методов: pooling, within, random.

Кроме того, в рамках модели Фамы - Френча методом бутстрап-симуляции определим, для каких фондов какой из факторов значим. Отдельно были протестированы однофакторные модели для $S M B$ и $H M L$.

\section{Результаты расчетов по гипотезе 1}

Практически все оцененные модели оказались незначимыми даже на 10\%-ном уровне значимости.

Из 599 фондов в выборке только у 21 фонда значимы все три фактора одновременно: «Капитал-Облигации плюс», «Две столицы», «Саларьево», «Рублевые облигации-2018», «Капитал-Депозитный», «КьюБиЭф Недвижимость», «РСХБ-Казначейский», «ИТ-сегмент», «Ритейл инвест», «Каскад Деметра», «Аршин», «Альтернативные инвестиции», «Технология роста», «Универсальные стратегии», «Энергетика Севера», «Финам-Депозитный», «Виктория», «Инвестбаланс», «Югра-Рантье», «РВМ», «Русский Транзит».

\section{Выводы по гипотезе 1}

Модель Фамы - Френча не обладает достаточной описательной силой в рамках российского рынка ПИФов (большинство моделей оказались в целом незначимыми), хотя несколько интересных особенностей все же можно выделить.

Во-первых, при тестировании модели Фамы - Френча в агрегированном виде коэффициент положителен и значим почти для всех типов открытых фондов, что говорит об оправданности рисков, взятых на себя менеджером фонда, и о значимом влиянии навыков управляющего на избыточную доходность в контексте размера и стоимости фонда. Однако при переходе к отдельным фондам он не оказался значимым ни в одном из случаев. Возможно, это связано с небольшим числом бутстрап-симуляций (их всего 50, хотя желательно хотя бы 1000). 
Bo-вторых, фактор размера оказывается значимым для фондов с низким риском инвестирования. Одно из возможных объяснений значимой положительной связи: будучи не расположенными к риску, инвесторы выбирают фонды такого типа, уделяя немаловажное значение его положению на рынке. Имея преимущество в объемах привлеченных средств и СЧА, у фондов больше возможностей выбора стратегий и получения избыточных доходностей. Значимый отрицательный коэффициент можно объяснить следующим образом. Небольшие по размеру фонды низкого риска «более аккуратны» при выборе стратегий и в итоге получают большую избыточную доходность, что позволяет им выделиться на фоне аналогичных по размеру фондов с более высокими рисками.

B-третьих, фактор стоимости оказался положительно значимым в случае закрытых и интервальных фондов высокого риска и практически равен нулю в случае открытых фондов низкого риска. Возможно, это связано с тем, что закрытые и интервальные ПИФы акций в основном вкладываются в более рисковые акции второго эшелона, что и улавливает коэффициент перед показателем $H M L$.

\section{Гипотеза 2: на российском рынке ПИФов присутствует моментум-эффект, и элементь портфельной моментум-стратегии влияют на результаты инвестирования.}

В рамках данной гипотезы проверяется тот факт, что при выборе инвестиционного фонда инвестор ориентируется на прошлые результаты деятельности фонда:

- $\quad$ на доходность прошлых периодов;

- $\quad$ на эффективность деятельности фонда прошлых периодов, оцененную такими общепринятыми показателями, как коэффициенты Шарпа, Трейнора, Сортино и т.д., которые, в свою очередь, позволяют учесть риск инвестиций.

Иными словами, проверяется, можно ли, формируя инвестиционный портфель на основании одного из двух вышеперечисленных критериев, сформировать правила отбора фондов таким образом, чтобы портфель с «нулевыми издержками» телей» или «проигравших» генерировал избыточную по отношению к выбранному бенчмарку прибыль.

\section{Результаты расчетов по гипотезе 2}

Получены средние результаты превышения избыточной доходности портфеля «победителей» над портфелем «проигравших» при разных окнах анализа $(3,6$, 9, 12 месяцев, Average с весами 1/4, и Weighted Average с весами 4/8, 2/8, 1/8, 1/8). Результаты сопоставления избыточных доходностей инвестирования в рамках двух сформированных портфелей («победителей» и «проигравших») показывают наличие краткосрочного моментум-эффекта (стратегия с периодов формирования в три месяца).

Таблица 6. Среднемесячные избыточные доходности портфелей Win, Los и WML (арбитражной стратегии) по семи стратегиям за период 2000-2016 гг.

\begin{tabular}{|c|c|c|c|}
\hline $\begin{array}{l}\text { ТиI } \\
\text { стратегии }\end{array}$ & Win & Los & $W M L$ \\
\hline \multirow{2}{*}{$12 \mathrm{M}$} & 0.0139 & 0.0171 & -0.0032 \\
\hline & $4.6397 * * *$ & $3.6685 * *$ & -0.8848 \\
\hline \multirow{2}{*}{$3 \mathrm{M}$} & 0.0169 & 0.0021 & 0.0148 \\
\hline & $5.1855 * * *$ & $3.6528 * *$ & $1.3439^{*}$ \\
\hline \multirow{2}{*}{$6 \mathrm{M}$} & 0.0145 & 0.0014 & 0.0131 \\
\hline & $4.7553 * * *$ & $3.4822 * *$ & 0.4024 \\
\hline \multirow{2}{*}{$9 \mathrm{M}$} & 0.0142 & 0.0180 & -0.0038 \\
\hline & $4.7386^{* * *}$ & $3.9243 * *$ & -0.0282 \\
\hline \multirow{2}{*}{ Average } & 0.0141 & 0.0124 & 0.0017 \\
\hline & $4.4986^{* * *}$ & $3.5755^{* *}$ & 0.6657 \\
\hline \multirow{2}{*}{$\begin{array}{l}\text { Weighted } \\
\text { Average }\end{array}$} & 0.0145 & 0.0004 & 0.0141 \\
\hline & $4.4777 * * *$ & $3.5991 * *$ & 1.0974 \\
\hline
\end{tabular}

Примечание: $t$-статистика представлена курсивом; уровни значимости: ${ }^{\star * *} 0-0.001 ;^{* *} 0-001-0.01$; *0.05-0.1-1.

Имеет место краткосрочный моментум-эффект - прибыльность стратегии $3 \mathrm{M}$ явно выделяется, в то время как избыточная доходность долгосрочной стратегии $12 \mathrm{M} \mathrm{уступает} \mathrm{всем} \mathrm{рассмотренным} \mathrm{вариантам.}$

В периоды спада в 2008-2009 и 2011-2012 гг. стратегия 3М принесла относительно меньше убытков. На протяжении 2013-2014 гг., наоборот, наилучшая производительность у стратегии $12 \mathrm{M}$, что можно объяснить надеждами инвесторов на краткосрочность экономического спада, полученным «опытом» в период кризиса 2008-2009 гг.

Дополнительно рассмотрена стратегия моментуминвестирования, скорректированная на риск. Для сравнения с простой стратегией $12 \mathrm{M}$ риск-скорректированные стратегии также применялись для окна 12 месяцев. Предварительно все коэффициенты были рассчитаны для окна в 30-31 день (по количеству дней в месяце).

\footnotetext{
${ }^{5}$ Портфель нулевых издержек (арбитражная стратегия) строится путем имитации действий инвестора, когда он занимает «длинную позицию» по портфелю прошлых победителей и «короткую» - по портфелю проигравших.
} 
Таблица 7. Среднемесячные избыточные доходности портфелей Win, Los и WML простой (12M) и риск-скорректированной моментум-стратегии

\begin{tabular}{|c|c|c|c|}
\hline Тип стратегии & Win («Победители») & Los («Проигравшие») & $W M L$ \\
\hline \multirow{2}{*}{ 12M (Simple) } & 0.0139 & 0.0171 & -0.0032 \\
\hline & $4.6397 * * *$ & $3.6685 * *$ & -0.8848 \\
\hline \multirow{2}{*}{ Sharpe Ratio Modified } & 0.0160 & 0.0159 & 0.0001 \\
\hline & $4.2296 * * *$ & $3.3069 * *$ & 0.0171 \\
\hline \multirow{2}{*}{ Sharpe Ratio } & 0.0182 & 0.0057 & 0.0094 \\
\hline & $1.8582 *$ & $1.7866^{*}$ & 1.2087 \\
\hline \multirow{2}{*}{ Treynor Ratio } & 0.0203 & 0.0187 & 0.0001 \\
\hline & $5.0424 * * *$ & $4.9498 * *$ & 1.5447 \\
\hline \multirow{2}{*}{ Sortino Ratio } & 0.0171 & 0.0159 & 0.0211 \\
\hline & $4.2296^{* * *}$ & $3.3069 * *$ & 0.0171 \\
\hline \multirow{2}{*}{ Modigliani Ratio } & 0.0136 & 0.015 & -0.0015 \\
\hline & $4.3878 * * *$ & $3.7042 * *$ & -0.4991 \\
\hline
\end{tabular}

Примечание: $t$-статистика представлена курсивом; уровни значимости: ${ }^{* \star *} 0-0.001 ;{ }^{* *} 0-001-0.01 ;{ }^{*} 0.05-0.1-1$.

В экономической литературе наиболее распространенным и общепринятым является расчет показателя $W M L$ модели Кархарта именно для периода формирования в 12 месяцев (аналогично первоисточнику [35]). В табл. 7 представлены средние результаты превышения доходности портфеля «победителей» над портфелем «проигравших» для различных критериев отбора фондов.

В качестве критерия отбора фондов лучше всего себя проявил коэффициент Трейнора, учитывающий систематический (рыночный) риск, выраженный коэффициентом $\beta$. Его доходность заметно превышает доходность простой моментум-стратегии с окном анализа в 12 месяцев.

Интересен тот факт, что в случае доходностей портфеля «победителей» корреляции коэффициентов положительны, хотя и наблюдается слабая связь между мерой Трейнора и остальными показателями. В то время как и в случае доходностей портфеля $W M L$ присутствует отрицательная зависимость между группой «простая стратегия 12M, мера Трейнора, коэффициент Модильяни» и группой «мера Шарпа, модифицированный коэффициент Шарпа, коэффициент Сортино». Такое поведение можно объяснить характером показателей, по которым они рассчитываются (коэффициенты в первой группе учитывают систематический риск, во второй - суммарный риск). Результаты тестирования однофакторной модели по моментуму для разных типов стратегий представлены в табл. 8.

Как следует из расчетов, для коэффициента Трейнора результаты значительно лучше, чем для других способов определения фактора моментума (из 380 фондов моментум положительно значим для 311 ПИФов).
Таблица 8. Результаты бутстрап-симулирования для однофакторных моделей (описательные статистики значений коэффициентов перед фактором)

\begin{tabular}{|c|c|c|c|c|}
\hline & \multicolumn{2}{|c|}{ Простой моментум } & \multicolumn{2}{|c|}{$\begin{array}{l}\text { Скорректированный } \\
\text { на риск моментум }\end{array}$} \\
\hline & $12 \mathrm{M}$ & $3 \mathrm{M}$ & $\begin{array}{l}\text { Treynor } \\
\text { Ratio }\end{array}$ & $\begin{array}{l}\text { Sharpe } \\
\text { Ratio }\end{array}$ \\
\hline Мин. знач. & 0.0058 & 0.0018 & 0.0035 & 0.0015 \\
\hline 1-й квантиль & 0.0236 & 0.0309 & 0.0703 & 0.0241 \\
\hline Медиана & 0.0735 & 0.0750 & 0.1709 & 0.0725 \\
\hline Ср. знач. & 0.1408 & 0.3536 & 0.3079 & 1.6096 \\
\hline 3-й квантиль & 0.2181 & 0.1601 & 0.2588 & 0.1278 \\
\hline Макс. знач. & 0.5292 & 7.8408 & 8.0973 & 56.7287 \\
\hline
\end{tabular}

Примечание: месячные данные за период с января 2000 по апрель 2016 г.

\section{Виводы по гипотезе 2:}

1) статистическая значимость моментум-эффекта на российском рынке ПИФов подтверждается в рамках краткосрочной арбитражной стратегии $(W M L)$ с окном анализа три месяца $(3 \mathrm{M})$ и на данных по 2013-2016 гг. - для долгосрочной стратегии 12M;

2) открытие инвестором «длинной позиции» по портфелям «победителей» в среднем значимо и прибыльно во всех рассмотренных случаях (максимальную доходность приносит в случае $3 \mathrm{M}$, на данных по 2013-2016 гг. - в случае 12M); 
3) рассматривая общепринятый в эмпирических исследованиях интервал формирования портфелей (12 месяцев), результаты существенно улучшает корректировка моментум-стратегии на риск (ориентирование на значения мер оценки эффективности фондов при формировании портфелей). Случай учета коэффициента Трейнора приносит самую высокую статистически значимую среднюю избыточную доходность из всех рассмотренных вариантов. Второй по доходности со статистически значимыми результатами - коэффициент Шарпа.

Иначе говоря, гипотеза о присутствии моментум-эффекта на российском рынке ПИФов и его влиянии на результаты инвестирования подтверждается.

Гипотеза 3: на избыточную доходность российских ПИФов влияют факторы размера, стоимости и моментума, в то же время коэффициент Кархарта статистически значим и может быть использован для дальнейшего анализа.

\section{Результать расчетов}

Тестирование модели Кархарта в агрегированном виде дало следующие результаты. Во-первых, при обычных методах проверки гипотез фактор моментума оказался положительно значим для выборки из всех открытых фондов. Во-вторых, коэффициент SMB оказался значимым только у открытых фондов облигаций, $H M L$ - у всех открытых фондов. Причем для обоих коэффициентов были значительные выбросы в случае интервальных фондов. В-третьих, ни одна из спецификаций модели не является значимой даже на 10\% уровне значимости (по критерию Фишера). По сравнению с моделью Фамы - Френча, которая оценивалась по дневным данным, результаты оценки модели Кархарта намного хуже.

При тестировании модели методом бутстрап для отдельных фондов можно выделить только 21 ПИФ со значимым на $10 \%$ уровне коэффициентом $\alpha$. Использовать а как скорректированную на риск избыточную доходность для всех фондов нецелесообразно.

\section{Гипотеза 4: на избыточную доходность российских ПИФов влияют микроэкономические характеристики фондов.}

\section{Результать расчетов}

Сравнение трех подходов к определению избыточной доходности указывает на относительно лучшее поведение модели с применением смешанной стратегии. Большее число факторов оказывается значимыми, значение скорректированного $R^{2}$ - самое высокое из трех рассмотренных случаев, и модель в целом почти значима на 10\%-ном уровне (табл. 9).

Таблица 9. Результаты тестирования модели с включенными микроэкономическими характеристиками фондов

\begin{tabular}{|c|c|c|c|}
\hline & \multicolumn{3}{|c|}{ Показатель избыточной доходности } \\
\hline & $\begin{array}{l}\text { a Кархарта } \\
\text { (для всех фондов) }\end{array}$ & $\begin{array}{c}\text { Избыточная относительно } \\
\text { бенчмарка доходность } \\
\text { (для всех фондов) }\end{array}$ & $\begin{array}{c}\text { Смешанная } \\
\text { стратегия }\end{array}$ \\
\hline СЧА фонда & 0.6738 & 1.9690 & 1.0688 \\
\hline $\begin{array}{l}\text { Тип фонда } \\
\text { (1 - открытый или интервальный, } 0 \text { - закрытый) }\end{array}$ & $1.1013 *$ & $0.0055 \%$ & $1.0209 * *$ \\
\hline $\begin{array}{l}\text { Объект инвестирования } \\
\text { (1 - высокого риска, } 0 \text { - низкого риска) }\end{array}$ & 0.0462 & -0.0939 & $0.2083 *$ \\
\hline $\begin{array}{l}\text { Количество дней после завершения } \\
\text { формирования фонда }\end{array}$ & $0.9381 * *$ & $1.7500 * *$ & $1.7590 * *$ \\
\hline Количество пайщиков & 0.0206 & 0.0086 & 0.0001 \\
\hline Минимальная сумма первого взноса & 0.0001 & 0.0069 & 0.0000 \\
\hline Минимальная сумма последующих взносов & 0.7430 & 0.4204 & 0.5865 \\
\hline $\begin{array}{l}\text { Надбавки УК } \\
\text { (сумма инвестиций до } 300 \text { тыс. руб.) }\end{array}$ & -0.3926 & -0.3514 & -0.3613 \\
\hline $\begin{array}{l}\text { Скидки УК } \\
\text { (срок инвестирования до одного года) }\end{array}$ & $0.3088 *$ & 0.3728 & 0.3765 \\
\hline Вознаграждения УК & -0.6194 & -0.6195 & -0.6195 \\
\hline Прочие расходы & $-0.0290 * *$ & $-0.0878 * * *$ & $-0.0799 * * *$ \\
\hline $\begin{array}{l}\text { Общий размер расходов, связанный } \\
\text { с деятельностью фонда }\end{array}$ & -1.3754 & -1.8035 & $2.7561 * *$ \\
\hline
\end{tabular}




\begin{tabular}{|c|c|c|c|}
\hline & \multicolumn{3}{|c|}{ Показатель избыточной доходности } \\
\hline & $\begin{array}{c}\text { a Кархарта } \\
\text { (для всех фондов) }\end{array}$ & $\begin{array}{l}\text { Избыточная относительно } \\
\text { бенчмарка доходность } \\
\text { (для всех фондов) }\end{array}$ & $\begin{array}{l}\text { Смешанная } \\
\text { стратегия }\end{array}$ \\
\hline Количество фондов в УК & 0.0000 & 0.0001 & $0.0001 *$ \\
\hline СЧА фондов УК & 0.4291 & 0.6221 & 0.4315 \\
\hline Доля по СЧА в управлении & 0.8774 & 0.3185 & 0.4737 \\
\hline Объемы привлеченных средств (за один год) & $1.1392 *$ & 0.8519 & $0.9158 *$ \\
\hline $\begin{array}{l}\text { Рейтинг УК от Национального рейтингового } \\
\text { агентства }\end{array}$ & 0.0001 & -0.0012 & 0.0001 \\
\hline $\operatorname{Adj} \cdot R^{2}$ & 0.0867 & 0.1295 & 0.2804 \\
\hline$F$-stat (p-value) & 0.4732 & 0.2892 & 0.1053 \\
\hline Уровень значимости: *** $0-0.001 ; * * 0-001-$ & $0.05-0.1-1$ & & \\
\hline
\end{tabular}

\section{Заключение}

Факторы размера и стоимости лучше оценивать по модели Фамы - Френча. В агрегированном виде фактор размера оказался значимым для фондов с низким уровнем риска, фактор стоимости - для высокорисковых интервальных и закрытых ПИФов акций. В случае рассмотрения отдельных фондов фактор стоимости значим для 40\% в выборке из 599 ПИФов, фактор стоимости - для 10\%. Для однофакторных моделей эти доли растут до 43 и 13\% соответственно. Для каждого фонда можно определить степень чувствительности доходности к данным факторам и направление влияния.

При выборе российских ПИФов можно ориентироваться на исторические данные (работает фактор моментума), что в среднем приносит статистически значимую положительную избыточную доходность. При этом в среднем оптимальным является период три месяца, и расчеты показывают, что в последние три года лучше себя проявляет долгосрочная стратегия в 12 месяцев, а потому нецелесообразно инвестировать, основываясь только на данных за длительные сроки. Корректировка на риск улучшает результаты, а лучше всего себя проявили меры Трейнора и Шарпа.

Оценка значимости коэффициента из модели Кархарта методом бутстрап позволяет выделить только 7\% из выборки в 380 ПИФов, для которых имеет смысл задавать избыточную доходность Кархарта.

При оценке микроэкономических факторов фондов целесообразно разделение выборки на ПИФы, для которых избыточная доходность задается коэффициентом альфа, и относительно бенчмарка (индекс ММВБ), что заметно улучшает получаемые результаты и повышает качество модели.

\section{Список литературы}

1. Абрамов А.Е., Акшенцева К.С., Чернова М.И., Логинова Д.А., Новиков Д.В., Радыгин А.Д. и др. Экономика инвестиционных фондов. М.: Дело; 2015. $720 \mathrm{c}$.

2. Bauer R., Otten R., Koedijk K. International evidence on ethical mutual fund performance and investment style. Journal of Banking \& Finance. 2005;29(7):17511767. DOI: 10.1016/j.jbankfin.2004.06.035

3. Hoepner A.G., Rammal H.G., Rezec M. Islamic mutual funds' financial performance and international investment style: Evidence from 20 countries. The European Journal of Finance. 2011;17(9-10):829-850. DOI: $10.1080 / 1351847 X .2010 .538521$

4. Frye M.B. The performance of bank-managed mutual funds. The Journal of Financial Research. 2001;24(3):419-442. DOI: 10.1111/j.1475-6803.2001. tb00778.x

5. Jain P.C., Wu J.S. Truth in mutual fund advertising: Evidence on future performance and fund flows. The Journal of Finance. 2000;55(2):937-958. DOI: 10.1111/0022-1082.00232

6. Kacperczyk M., Sialm C., Zheng L. Unobserved actions of mutual funds. The Review of Financial Studies. 2008;21(6):2379-2416. DOI: 10.1093/rfs/ hhl041

7. Sensoy B.A. Performance evaluation and selfdesignated benchmark indexes in the mutual fund industry. Journal of Financial Economics. 2009;92:2539. DOI:10.1016/j.jfineco.2008.02.011 
8. Hansen H.H., Haukaas E.S., Gallefoss K. Performance and persistence in Norwegian mutual funds. Trondheim: Norwegian University of Science and Technology; 2012. URL: https://brage.bibsys.no/ xmlui/bitstream/handle/11250/266269/629181_ FULLTEXT01.pdf? sequence $=2 \&$ isAllowed $=\mathrm{y}$

9. Hoang N. Swedish Hedge Fund and mutual fund performance during the financial crisis of 2008. Gothenburg: School of Business, Economics and Law, University of Gothenburg; 2015. 38 p. URL: https:/gupea.ub.gu.se/bitstream/2077/40543/1/ gupea_2077_40543_1.pdf

10. Теплова Т.В. Моментум-эффект на рынке акций и инвестиционная торговая стратегия "По течению”: методики тестирования и развитие модели ценообразования финансовых активов. Управление финансовыми рисками. 2013;(4):282-295.

11. Абрамов А.Е., Акшенцева К.С. Развитие взаимных фондов в России. Экономическая политика. 2014;(1):35-53.

12. Абрамов А.Е., Акшенцева К.С., Радыгин А.Д. Эффективность паевых инвестиционных фондов: теоретические подходы и опыт России. Экономическая политика. 2015;10(4):60-86. DOI: 10.18288/1994-5124-2015-4-04

13. Jank S., Wedow M. Purchase and redemption decisions of mutual fund investors and the role of fund families. The European Journal of Finance. 2013;19(2):127-144. DOI: 10.1080/1351847X.2012.662908

14. Warner J.B., Wu J.S. Why do mutual fund advisory contracts change? Performance, growth, and spillover effects. The Journal of Finance. 2011;66(1):271-306. DOI: $10.1111 /$ j.1540-6261.2010.01632.x

15. Chen J., Hong H., Huang M., Kubik J. Does fund size erode mutual fund performance? The role of liquidity and organization. American Economic Review. 2004;94(5):1276-1302. DOI: 10.2139/ssrn.372721

16. Ferreira M.A., Keswani A., Miguel A., Ramos S.B. The determinants of mutual fund performance: A crosscountry study. Review of Finance. 2013;17(2):483-525. DOI: $10.1093 /$ rof/rfs013

17. Gruber M. Another puzzle: The growth in actively managed mutual funds. The Journal of Finance. 1996;51(3):783-810. DOI: 10.1111/j.1540-6261.1996. tb02707.x

18. Zheng L. Is money smart? A study of mutual fund investors' fund selection ability. The Journal of Finance. 1999;54(3):901-933. DOI: 10.1111/00221082.00131

19. Sapp T., Tiwari A. Does stock return momentum explain the "smart money" effect? The Journal of Finance. 2004;59(6):2605-2622. DOI: 10.1111/j.15406261.2004.00710.x
20. Gil-Bazo J., Ruiz-Verdú P. Yet another puzzle? The relation between price and performance in the mutual fund industry. The Journal of Finance. 2009;64(5):2153-2183. DOI: 10.1111/j.15406261.2009.01497.x

21. Droms W.G., Walker D.A. Mutual fund investment performance. The Quarterly Review of Economics and Finance. 1996;36(3):347-363.

22. Yuan R., Xiao J.Z., Zou X. Mutual funds' ownership and firm performance: Evidence from China. Journal of Banking and Finance. 2008;32(8):1552-1565. DOI: 10.1016/j.jbankfin.2007.08.001

23. Kosowski R., Timmermann A., Wermers R., White H. Can mutual fund "stars" really pick stocks? New evidence from a bootstrap analysis. The Journal of Finance. 2006;61(6):2551-2595. DOI: 10.1111/j.15406261.2006.01015.x

24. Паршаков П.А. Оценка эффективности деятельности по управлению активами российских паевых инвестиционных фондов. Дис. ... канд. экон. наук. М.: НИУ ВШЭ; 2014. 184 с.

25. Муравьев Д.М. Обыгрывают ли российские паевые фонды рынок? Российская экономическая школа. Препринт. 2006;(085 R). URL: https:// docplayer.ru/27522171-Muravev-d-m-obygryvayutli-rossiyskie-paevye-fondy-rynok.html

26. Blake D., Ioannidis C., Caulfield T., Tonks I. Improved inference in the evaluation of mutual fund performance using panel bootstrap methods. Journal of Econometrics. 2014;183(2):202-210. DOI: 10.1016/j.jeconom.2014.05.010

27. Ippolito R. Consumer reaction to measures of poor quality: Evidence from the mutual fund industry. The Journal of Law and Economics. 1992;35(1):45-70. DOI: $10.1086 / 467244$

28. Lynch A., Musto D. How investors interpret past fund returns. The Journal of Finance. 2003;58(5):20332058. DOI: $10.1111 / 1540-6261.00596$

29. Ingersoll J., Spiegel M., Goetzmann W., Welch I. Portfolio performance manipulation and manipulation-proof performance measures. The Review of Financial Studies. 2007;20(5):1503-1546. DOI: $10.1093 / \mathrm{rfs} / \mathrm{hhm} 025$

30. Moore O. Mutual fund age, performance, and the optimal track record. Stanford, CA: Department of Economics, Stanford University; 2016. 47 p. URL: https://economics.stanford.edu/sites/default/files/ publications/olivia_moore_honors_thesis.pdf

31. Grinblatt M., Titman S. A Study of monthly mutual fund returns and performance evaluation techniques. The Journal of Financial and Quantitative Analysis. 1994;29(3):419-444.

DOI: $10.2307 / 2331338$ 
32. Elton E., Gruber M., Blake C. Incentive fees and mutual funds. The Journal of Finance. 2003;58(2):779804. DOI: 10.1111/1540-6261.00545

33. Dewri L., Islam M.R. Performance of public mutual funds (PMFs) in emerging economies: A case of Bangladesh. International Journal of Business and Management. 2016;11(6):296-301. DOI: 10.5539/ ijbm.v11n6p296\}
34. Володин С.Н., Кузнецова М.С.

Сравнение активно и пассивно управляемых инвестиционных фондов. Корпоративные финансы. 2015;9(4):89-102.

DOI: 10.17323/j.jcfr.2073-0438.9.4.2015.88-101

35. Carhart M.M. On persistence in mutual fund performance. The Journal of Finance. 1997;52(1):5782. DOI: $10.2307 / 2329556$ 


\title{
Determinants of Mutual Funds Performance
}

\author{
Alexandra Galanova \\ $\mathrm{PhD}$, Associate Professor of the School of Finance \\ National Research University Higher School of Economics \\ 20 Myasnitskaya St, Moscow, Russia \\ E-mail: agalanova@hse.ru \\ Valeriia Dukova \\ Research Intern \\ National Research University Higher School of Economics \\ 20 Myasnitskaya St, Moscow, Russia \\ E-mail: vdukova@hse.ru
}

\begin{abstract}
This article proposes a new extended approach to identifying the economic determinants of the performance of Russian mutual funds. The purpose of this study is to identify the factors that affect mutual funds' excess return, both in the aggregate form (for the Russian mutual fund market in general, i.e. broad categories of funds with different underlying assets types), and for specific mutual funds (i.e. the specific performance indicators of individual funds). The conventional analysis scheme is extended by two stages: first, by analysing the momentum-effect (simple and risk-adjusted) using the Carhart model; and secondly, by testing the hypothesis on the coefficients significance using the bootstrap-method, which allows one to analyse the excess return of specific, individual mutual funds. Data from a sample of 667 Russian mutual funds for the period from 2000 to 2016 was analysed. The study revealed the following results. Investors can rely on historical data when selecting Russian mutual funds (the momentum factor is significant). Adjustment for risk improves the results (Treynor and Sharpe measures seem to cope best of all). The a Carhart coefficient is significant for only $7 \%$ of the sample, and thus it does not make sense to employ it in further analysis for every fund. The excess returns in the regression with funds' characteristics are suitable to be set apart by the a Carhart coefficient and the benchmark estimation, which significantly refines the obtained results and improves the quality of the model.
\end{abstract}

Keywords: mutual fund, excess return, benchmark, investment performance criteria, risk, momentum effect, bootstrap method

JEL: G11, G24, G32, G34 


\section{References}

1. Abramov A.E., Akshentseva K.S., Chernova M.I., Loginova D.A., Novikov D.V., Radygin A.D. et al. Economics of investment funds. Moscow: Delo; 2015. 720 p. (In Russ.).

2. Bauer R., Otten R., Koedijk K. International evidence on ethical mutual fund performance and investment style. Journal of Banking \& Finance. 2005;29(7):17511767. DOI: 10.1016/j.jbankfin.2004.06.035

3. Hoepner A.G., Rammal H.G., Rezec M. Islamic mutual funds' financial performance and international investment style: Evidence from 20 countries. The European Journal of Finance. 2011;17(9-10):829-850. DOI: $10.1080 / 1351847 X .2010 .538521$

4. Frye M.B. The performance of bank-managed mutual funds. The Journal of Financial Research. 2001;24(3):419-442. DOI: 10.1111/j.1475-6803.2001. tb00778.x

5. Jain P.C., Wu J.S. Truth in mutual fund advertising: Evidence on future performance and fund flows. The Journal of Finance. 2000;55(2):937-958. DOI: 10.1111/0022-1082.00232

6. Kacperczyk M., Sialm C., Zheng L. Unobserved actions of mutual funds. The Review of Financial Studies. 2008;21(6):2379-2416. DOI: 10.1093/rfs/ hhl041

7. Sensoy B.A. Performance evaluation and selfdesignated benchmark indexes in the mutual fund industry. Journal of Financial Economics. 2009;92:2539. DOI:10.1016/j.jfineco.2008.02.011

8. Hansen H.H., Haukaas E.S., Gallefoss K. Performance and persistence in Norwegian mutual funds. Trondheim: Norwegian University of Science and Technology; 2012. URL: https://brage.bibsys.no/ xmlui/bitstream/handle/11250/266269/629181_ FULLTEXT01.pdf? sequence $=2$ \&isAllowed $=y$

9. Hoang N. Swedish Hedge Fund and mutual fund performance during the financial crisis of 2008. Gothenburg: School of Business, Economics and Law, University of Gothenburg; 2015. 38 p. URL: https:/gupea.ub.gu.se/bitstream/2077/40543/1/ gupea_2077_40543_1.pdf

10. Teplova T.V. Momentum-effect in the stock market and the investment trading strategy "Downstream": Testing techniques and the development of the pricing model for financial assets Upravlenie finansovymi riskami. 2013;(4):282-295. (In Russ.).

11. Abramov A.E., Akshentseva K.S. Development of mutual funds in Russia. Ekonomicheskaya politika = Economic Policy. 2014;(1):35-53. (In Russ.).

12. Abramov A.E., Akshentseva K.S., Radygin A.D.
Effectiveness of mutual funds: Theoretical approaches and experience of Russia. Ekonomicheskaya politika = Economic Policy. 2015;10(4):60-86. DOI: 10.18288/1994-5124-2015-4-04 (In Russ.).

13. Jank S., Wedow M. Purchase and redemption decisions of mutual fund investors and the role of fund families. The European Journal of Finance. 2013;19(2):127-144. DOI: 10.1080/1351847X.2012.662908

14. Warner J.B., Wu J.S. Why do mutual fund advisory contracts change? Performance, growth, and spillover effects. The Journal of Finance. 2011;66(1):271-306. DOI: $10.1111 / \mathrm{j} .1540-6261.2010 .01632 . \mathrm{x}$

15. Chen J., Hong H., Huang M., Kubik J. Does fund size erode mutual fund performance? The role of liquidity and organization. American Economic Review. 2004;94(5):1276-1302. DOI: 10.2139/ssrn.372721

16. Ferreira M.A., Keswani A., Miguel A., Ramos S.B. The determinants of mutual fund performance: A crosscountry study. Review of Finance. 2013;17(2):483-525. DOI: $10.1093 / \mathrm{rof} / \mathrm{rfs} 013$

17. Gruber M. Another puzzle: The growth in actively managed mutual funds. The Journal of Finance. 1996;51(3):783-810. DOI: 10.1111/j.1540-6261.1996. tb02707.x

18. Zheng L. Is money smart? A study of mutual fund investors' fund selection ability. The Journal of Finance. 1999;54(3):901-933. DOI: 10.1111/00221082.00131

19. Sapp T., Tiwari A. Does stock return momentum explain the "smart money" effect? The Journal of Finance. 2004;59(6):2605-2622. DOI: 10.1111/j.15406261.2004.00710.x

20. Gil-Bazo J., Ruiz-Verdú P. Yet another puzzle? The relation between price and performance in the mutual fund industry. The Journal of Finance. 2009;64(5):2153-2183. DOI: 10.1111/j.15406261.2009.01497.x

21. Droms W.G., Walker D.A. Mutual fund investment performance. The Quarterly Review of Economics and Finance. 1996;36(3):347-363.

22. Yuan R., Xiao J.Z., Zou X. Mutual funds' ownership and firm performance: Evidence from China. Journal of Banking and Finance. 2008;32(8):1552-1565. DOI: 10.1016/j.jbankfin.2007.08.001

23. Kosowski R., Timmermann A., Wermers R., White H. Can mutual fund "stars" really pick stocks? New evidence from a bootstrap analysis. The Journal of Finance. 2006;61(6):2551-2595. DOI: 10.1111/j.15406261.2006.01015.x

24. Parshakov P.A. Evaluation of the effectiveness of asset management of Russian mutual funds. Cand. econ. sci. diss. Moscow: NRU HSE; 2014. 184 p. 
25. Murav'ev D. M. Do Russian mutual funds beat the market? New Economic School. Preprint. 2006;(085 R). URL: https://docplayer.ru/27522171-Muravevd-m-obygryvayut-li-rossiyskie-paevye-fondy-rynok. html (In Russ.).

26. Blake D., Ioannidis C., Caulfield T., Tonks I. Improved inference in the evaluation of mutual fund performance using panel bootstrap methods. Journal of Econometrics. 2014;183(2):202-210. DOI: 10.1016/j.jeconom.2014.05.010

27. Ippolito R. Consumer reaction to measures of poor quality: Evidence from the mutual fund industry. The Journal of Law and Economics. 1992;35(1):45-70. DOI: $10.1086 / 467244$

28. Lynch A., Musto D. How investors interpret past fund returns. The Journal of Finance. 2003;58(5):20332058. DOI: 10.1111/1540-6261.00596

29. Ingersoll J., Spiegel M., Goetzmann W., Welch I. Portfolio performance manipulation and manipulation-proof performance measures. The Review of Financial Studies. 2007;20(5):1503-1546. DOI: $10.1093 / \mathrm{rfs} / \mathrm{hhm} 025$

30. Moore O. Mutual fund age, performance, and the optimal track record. Stanford, CA: Department of Economics, Stanford University; 2016. 47 p. URL: https:/economics.stanford.edu/sites/default/files/ publications/olivia_moore_honors_thesis.pdf
31. Grinblatt M., Titman S. A Study of monthly mutual fund returns and performance evaluation techniques. The Journal of Financial and Quantitative Analysis. 1994;29(3):419-444. DOI: 10.2307/2331338

32. Elton E., Gruber M., Blake C. Incentive fees and mutual funds. The Journal of Finance. 2003;58(2):779804. DOI: 10.1111/1540-6261.00545

33. Dewri L., Islam M.R. Performance of public mutual funds (PMFs) in emerging economies: A case of Bangladesh. International Journal of Business and Management. 2016;11(6):296-301. DOI: 10.5539/ ijbm.v11n6p296\}

34. Volodin S.N., Kuznetsova M.S. The comparison of actively and passively managed investment funds. Korporativnye finansy = Journal of Corporate Finance Research. 2015;9(4):89-102. DOI: 10.17323/j. jcfr.2073-0438.9.4.2015.88-101 (In Russ.).

35. Carhart M.M. On persistence in mutual fund performance. The Journal of Finance. 1997;52(1):5782. DOI: $10.2307 / 2329556$ 